\title{
Desempenho zootécnico de alevinos de tilápia do Nilo cultivados em um sistema de recirculação de água com a microalga Spirulina platensis
}

\author{
Growth performance of Nile tilapia cultured in a recirculating water system with \\ microalgae "Spirulina platensis"
}

\author{
COÊLHO, Anderson Alan da Cruz ${ }^{1 *}$; BEZERRA, João Henrique Cavalcante ${ }^{2}$; SILVA, \\ José William Alves da ${ }^{3}$; MOREIRA, Renato Teixeira ${ }^{1}$; ALBUQUERQUE, Leonardo \\ Freitas Galvão de ${ }^{1}$; FARIAS, Wladimir Ronald Lobo ${ }^{4}$
}

\footnotetext{
${ }^{1}$ Instituto Federal do Ceará, Morada Nova, Ceará, Brasil.

${ }^{2}$ Instituto Centro de Ensino Tecnológico, Guaiúba, Ceará, Brasil.

${ }^{3}$ Instituto Federal do Ceará, Limoeiro do Norte, Ceará, Brasil.

${ }^{4}$ Universidade Federal do Ceará, Fortaleza, Ceará, Brasil

*Endereço para correspondência: anderson.coelho@ifce.edu.br
}

RESUMO

Objetivou-se com este estudo avaliar os parâmetros zootécnicos das tilápias do Nilo, qualidade de água, e taxa de remoção dos compostos nitrogenados pela microalga Spirulina platensis em um sistema fechado de circulação de água. $\mathrm{O}$ experimento foi realizado no Centro de Biotecnologia Aplicada a Aquicultura (CEBIAQUA) do Departamento de Engenharia de Pesca da Universidade Federal do Ceará. O cultivo das tilápias foi inteiramente casualisado e realizado em três tratamentos com quatro repetições nas densidades de 0,$1 ; 0,15$ e 0,20 alevinos $\mathrm{L}^{-1}$. As tilápias foram alimentadas ad libitum com ração comercial $(0.8 \mathrm{~mm})$, contendo $55 \%$ de proteína bruta $(\mathrm{PB})$, fornecida às $9 \mathrm{~h} 00 \mathrm{~min}, 13 \mathrm{~h} 00 \mathrm{~min} \mathrm{e}$ 17h00min, sete dias por semana. Durante todo o experimento, a água e das culturas da tilápia do Nilo e da $S$. platensis foi recirculada, havendo apenas reposição de água por conta da evaporação. Os resultados mostraram que a densidade de estocagem mais eficiente para o cultivo das tilápias foi 0,10 alevinos $\mathrm{L}^{-1}$, uma vez que apresentou a menor taxa de conversão alimentar. Com relação à qualidade de água, os níveis de amônia $(0,26 \pm$ $0,03 \mathrm{mg}$ L-1), nitrato $(2,55 \pm 0,71)$ e nitrito $(0,19 \pm$ $0,01)$ permaneceram dentro dos valores aceitáveis para o cultivo da tilápia do Nilo. Além disso, foram reduzidos $95,57 \pm 1,1 \%$ da amônia, 87,9 \pm $4,4 \%$ de nitrato e $42,63 \pm 8,5 \%$ de nitrito da água de cultivo dos peixes.

Palavras-chave: biorremediação, recirculação, sustentabilidade

\section{SUMMARY}

The present work aimed to evaluate the zootechnical parameters of Nile tilapia, water quality and rate of nitrogen removal compounds by Spirulina platensis in a closed system of water circulation. The experiment took place at the Biotechnology Aquaculture Applied Center (CEBIAQUA) of The Fishing Engineering Department of the Federal University of Ceará. Tilapias culture was conducted in three treatments with four replicates at densities of $010,0,15$ and 0,20 juveniles $\mathrm{L}^{-1}$. The tilapias were fed ad libitum with a commercial ration $(0,8 \mathrm{~mm})$ containing $55 \%$ crude protein $(\mathrm{CP})$, administered daily at 9,13 and $17 \mathrm{~h}$. Throughout the experiment, the water of fishes boxes and of the respective microalgae basins was reused in the closed recirculation system between the two cultures, with only replacement of water due to evaporation. The results showed that the most efficient stocking density was 0,10 $\mathrm{L}^{-1}$, because it presented the lowest feed conversion ratio. With respect to water quality, levels of $\mathrm{N}$-ammonia $\left(0,26 \pm 0,03 \mathrm{mg} \mathrm{L}^{-1}\right), \mathrm{N}$ nitrates $\left(2,55 \pm 0,71 \mathrm{mg} \mathrm{L}^{-1}\right)$ and $\mathrm{N}$ - nitrites $(0,19$ $\left.\pm 0,01 \mathrm{mg} \mathrm{L}^{-1}\right)$ remained within acceptable values for the cultivation of tilapia Nile. Moreover, were reduced $95.57 \pm 1.1 \%$ of ammonia, $87.9 \pm 4.4 \%$ of nitrate and $42.63 \pm$ $8.5 \%$ of nitrite of water fish culture.

Keywords: bioremediation, recirculation, sustainability 


\section{INTRODUÇ̃̃̃O}

A produção de organismos em cativeiro tem aumentado significantemente em todo mundo. Das 145,1 milhões de toneladas produzidas em 2009, 117,8 milhões tiveram como destino 0 consumo humano, sendo 55,1 milhões de toneladas $(46 \%)$ oriundas da aquicultura, o que contribui para o aumento do consumo (FAO, 2010).

No entanto, segundo Jones et al. (2002) o elevado crescimento na produção de organismos aquáticos tem afetado seriamente os ecossistemas aquáticos. Dessa forma, para que a produção continue em crescimento, uma das alternativas é o desenvolvimento de novas tecnologias que utilizem menos espaço e produzam o mínimo de impactos ambientais.

Esforços para o desenvolvimento de novas técnicas de cultivo mais sustentáveis na aquicultura têm sido realizado devido aos potenciais impactos negativos da atividade. Uma destas técnicas é a fitorremediação que utiliza vegetais aquáticos, como as algas, para assimilar os nutrientes produzidos pelo cultivo de animais aquáticos (ARANA, 2004). As algas podem absorver grandes quantidades de nutrientes dissolvidos (orgânicos e inorgânicos), geralmente com preferência para a amônia (PORRELLO et al., 2003; LOURENÇO, 2006).

Uma das espécies componentes do fitoplâncton que pode ser utilizada como fitorremediadora de efluentes da aquicultura intensiva é a Spirulina (Arthrospira) platensis, uma cianofícea com um elevado teor de proteína, nutrientes essenciais e compostos antioxidantes (COLLA et al., (2007).

A $S$. platensis necessita de água com sais minerais e $\mathrm{pH}$ elevado, luz e temperaturas altas. Essas condições para sua produção são perfeitamente garantidas pelo clima do semi-árido nordestino: luz o ano todo, praticamente sem variabilidade de incidência de raios solares de janeiro a dezembro, garantido uma temperatura, também, bastante estável o ano inteiro (LOURENÇO, 2006).

O presente trabalho teve como objetivos avaliar o desempenho zootécnico de alevinos de tilápia do Nilo, avaliar a evolução dos parâmetros de qualidade de água qualidade de água bem como a eficiência da remoção de compostos nitrogenados dos efluentes provenientes do cultivo de alevinos de tilápia do Nilo pela microalga S. platensis.

\section{MATERIAL E MÉTODOS}

As de Tilápia do Nilo já revertidas sexualmente e com peso médio de $1,0 \pm$ $0,5 \mathrm{~g}$, foram obtidas na Estação de Piscicultura Prof. Dr. Raimundo Saraiva da Costa do Departamento de Engenharia de Pesca da Universidade Federal do Ceará (UFC), e transportadas em recipientes de 50L providos de aeração constante, para a unidade de produção no Centro de Biotecnologia Aplicada à Aquicultura (CEBIAQUA) do mesmo Departamento.

Antes do início do cultivo, os peixes passaram por um período de adaptação a salinidade $10 \mathrm{ppt}$ por 24 horas, para equiparar com a salinidade dos cultivos de S. platensis.

Foram realizados três tratamentos com quatro repetições, em um delineamento inteiramente casualisado em 29 dias de cultivo. Os tratamentos foram diferenciados quanto a densidade de cultivo de $0,10,0,15$ e 0,20 peies $\mathrm{L}^{-1}$. Para isso, foram utilizados 12 tanques quadrangulares $(22 \times 45 \times 40 \mathrm{~cm})$ de polietileno com volume útil de 40L. Os 
tanques foram cobertos com tampa para sombrear os cultivos, e assim evitar o crescimento de outras microalgas no cultivo dos peixes e todos foram submetidos a aeração.

Posteriormente, durante o período de cultivo, foram alimentados com ração comercial $(0.8 \mathrm{~mm})$ com $55 \%$ de proteína bruta (PB), fornecida até a saciedade aparente diariamente três vezes ao dia às 09h00min, 13h00min e $17 \mathrm{~h} 00 \mathrm{~min}$, com exceção dos dias das biometrias, as quais ocorreram quinzenalmente. Para isto, Os peixes de cada tanque foram pesados em balança digital com precisão de $0,1 \mathrm{~g}$ para a determinação dos seguintes parâmetros zootécnicos: Taxa de Sobrevivência ( $\mathrm{S} \%)$ (SOUZA et al., 2008), Ganho de Peso (GP) (MARQUES et al., 2004), Ganho de Peso Médio Diário (GPD) (SILVA et al., 2006), Taxa de Crescimento Específico (TCE) (ASADUZZAMAN et al., 2009), e Taxa de Conversão Alimentar Aparente (CAA) (SANTOS et al., 2009).

Após 15 dias e no final do experimento, $50 \%$ da água de cultivos dos peixes (efluente) foi sifonada para tanques extras, posteriormente, o mesmo volume de água dos cultivos de $S$. platensis foi filtrada em telas de $60 \mu \mathrm{m}$ no interior dos tanques de tilápia. Por fim, os efluentes presentes nos tanques extras foram bombeados para os tanques de cultivo de $S$. platensis. Não houve renovação de água durante todo período experimental, apenas reposição das perdas de água por evaporação.

Após a recirculação da água, o percentual de remoção (R) dos compostos nitrogenados após a recirculação para cada tratamento foi calculado pela equação $\mathrm{R}=100$ - $(100 \times$ concentração dos compostos nitrogenados no efluente após a recirculação) / concentração dos compostos nitrogenados no efluente antes da recirculação, conforme descrita por Henry-Silva \& Camargo (2008).
Diariamente, as 09h00min, os parâmetros físicos e químicos da água de cultivo dos peixes foram monitorados, sendo determinado oxigênio dissolvido (mg $\mathrm{L}^{-}$ $\left.{ }^{1}\right), \mathrm{pH}$, temperatura $\left({ }^{\circ} \mathrm{C}\right)$ e salinidade (ppt). As concentrações de amônia (mg L$\left.{ }^{1}\right)$, nitrito $\left(\mathrm{mg} \mathrm{L}^{-1}\right)$ e nitrato $\left(\mathrm{mg} \mathrm{L}^{-1}\right)$ foram monitorados antes $\mathrm{e}$ após $\mathrm{O}$ fechamento de cada recirculação de água.

$\mathrm{O} \mathrm{pH}$ foi determinado com um medidor de $\mathrm{pH}$ de bancada (HANNA HI 221), a temperatura e o oxigênio dissolvido com uma sonda eletrônica (YSI 55) e a salinidade foi avaliada com um refratômetro (ATAGO). As concentrações de N-amônia (NH3), Nnitrato (NO2-) e N-nitrito (NO3-) foram determinadas por espectrofotometria de leitura direta em espectrofotômetro (HACH DR 2000).

Os resultados dos parâmetros zootécnicos dos peixes, dos parâmetros de qualidade de água e da taxa de remoção dos compostos nitrogenados nos diferentes tratamentos foram submetidos às análises de variância (ANOVA) simples $(\mathrm{p}<0,05)$ e no caso de diferenças significativas, as médias dos tratamentos foram comparadas pelo teste de Tukey $(\mathrm{p}<0,05)$.

\section{RESULTADOS E DISCUSSÃO}

Para a maioria dos parâmetros zootécnicos houve diferença estatística entre as três densidades experimentadas $(p<0,05)$. Apenas a biomassa final e $o$ ganho de biomassa não foram diferentes $(\mathrm{p}>0,05)$ entre os tratamentos (Tabela 1). Os peixes na densidade de 0,10 alevinos $\mathrm{L}^{-1}$ foram os que obtiveram melhores valores para $(p<0,05)$ o peso médio final, ganho de peso médio diário, taxa de crescimento específico, ganho de peso relativo e taxa de conversão alimentar. Pode-se observar 
que este tratamento se consolidou como sendo o mais eficiente nestas condições de cultivo. Mesmo a biomassa final sendo a mesma entre os tratamentos $(\mathrm{p}>0,05)$, a quantidade de alimento fornecido ao tratamento com a menor densidade de estocagem foi inferior aos demais $(\mathrm{p}<0,05)$.

Tabela 1. Desempenho das culturas de tilápia do Nilo cultivada com densidades de 0,10 , 0,15 e 0,20 alevinos $\mathrm{L}^{-1}$ durante o período de cultivo

\begin{tabular}{|c|c|c|c|}
\hline \multirow{2}{*}{ Parâmetros } & \multicolumn{3}{|c|}{ Tratamento (alevinos $\mathrm{L}^{-1}$ ) } \\
\hline & 0,10 & 0,15 & 0,20 \\
\hline Sobrevivência (\%) & $85,00 \pm 10,00^{\mathrm{ab}}$ & $95,83 \pm 8,33^{\mathrm{a}}$ & $71,88 \pm 6,25^{b}$ \\
\hline Peso médio inicial (g peixe ${ }^{-1}$ ) & $1,00 \pm 0,5^{\mathrm{a}}$ & $1,00 \pm 0,5^{\mathrm{a}}$ & $1,00 \pm 0,5^{\mathrm{a}}$ \\
\hline Biomassa inicial (g tanque ${ }^{-1}$ ) & $4,0 \pm 1,0^{\mathrm{b}}$ & $6,0 \pm 1,0^{\mathrm{ab}}$ & $8,0 \pm 1,0^{\mathrm{a}}$ \\
\hline Peso médio final ( $\mathrm{g}$ tanque ${ }^{-1}$ ) & $7,68 \pm 0,92^{\mathrm{a}}$ & $5,92 \pm 0,71^{\mathrm{b}}$ & $5,30 \pm 0,74^{\mathrm{b}}$ \\
\hline Biomassa final $\left(\mathrm{g}\right.$ tanque $\left.^{-1}\right)$ & $32,35 \pm 2,20^{\mathrm{a}}$ & $33,95 \pm 4,50^{\mathrm{a}}$ & $30,25 \pm 2,43^{\mathrm{a}}$ \\
\hline Ganho de peso médio diário $\left(\mathrm{g}\right.$ dia $\left.^{-1}\right)$ & $0,23 \pm 0,02^{\mathrm{a}}$ & $0,17 \pm 0,02^{\mathrm{b}}$ & $0,14 \pm 0,03^{\mathrm{b}}$ \\
\hline Taxa de crescimento específico $\left(\%\right.$ dia $\left.^{-1}\right)$ & $1,82 \pm 0,11^{\mathrm{a}}$ & $1,49 \pm 0,17^{\mathrm{b}}$ & $1,34 \pm 0,19^{\mathrm{b}}$ \\
\hline Ganho de biomassa $(\mathrm{g})$ & $26,85 \pm 2,20^{\mathrm{a}}$ & $27,35 \pm 4,50^{\mathrm{a}}$ & $22,55 \pm 2,43^{\mathrm{a}}$ \\
\hline Taxa de conversão alimentar $\left(\mathrm{g} \mathrm{g}^{-1}\right)$ & $1,72 \pm 0,25^{\mathrm{b}}$ & $2,8 \pm 0,46^{\mathrm{b}}$ & $3,22 \pm 0,56^{\mathrm{a}}$ \\
\hline
\end{tabular}

*Os valores representam as médias e desvio padrão dos parâmetros zootécnicos.

Letras diferentes representam diferença estatística $(\mathrm{p}<0,05)$.

Apesar da maioria dos parâmetros terem permanecido dentro da faixa tolerável para a espécie, a redução do peso médio e do ganho de peso nas densidades de 0,15 e 0,20 alevinos $\mathrm{L}^{-3}$ pode ser atribuída à maior densidade de estocagem, pois a densidade de estocagem é pode interferir diretamente no crescimento, na eficiência alimentar e na sobrevivência, além de interferir no comportamento dos peixes (SALARO et al., 2003).

A taxa de sobrevivência foi mais alta nos tratamentos com 0,10 e 0,15 alevinos $\mathrm{L}^{-1}(\mathrm{p}<0,05)$, o que evidencia também a elevada densidade de estocagem como sendo a causa da menor eficiência do tratamento com a maior densidade. Esses valores foram semelhantes aos encontrados por Bailey et al. (2000), que relataram taxas de sobrevivência de 91,2 a 91,9\%, sem diferenças entre cultivos de tilápias com densidades de 200 e 450 alevinos $\mathrm{m}^{-3}$ em um sistema de recirculação de água. Para melhor avaliar o desempenho dos animais foram utilizadas as taxas de crescimento específica $\left(\begin{array}{lll}\% & \left.\mathrm{dia}^{-1}\right) & \text { dos }\end{array}\right.$ animais nas três diferentes densidades de estocagem. Os valores do presente experimento foram diferentes de outros trabalhos, como o de El-sayed (2002) que, avaliando o efeito de diferentes densidades de estocagens de tilápias $\left(3,0 ; 5,0 ; 10,0 ; 15,0 ;\right.$ e 20,0 alevinos $\left.\mathrm{L}^{-3}\right)$ em tanques de fibra de vidro, observou que a TCE diminuiu com aumento da densidade populacional decrescendo de 10,05 a $7,78 \% \mathrm{dia}^{-1}$, valores bem superiores aos encontrados neste trabalho.

Silva et al. (2002) encontraram taxas de crescimento específico mais elevadas que as observadas no presente experimento, quando avaliaram o desempenho produtivo de alevinos de tilápia do Nilo (Oreochromis niloticus) estocados nas densidades de $0,18,0,24$ e 0,30 alevinos $\mathrm{L}^{-3}$ em tanques circulares, porém com duas trocas totais de água (30 e 60 minutos) em um sistema de "raceway". Eles obtiveram TCE de 2,30, 2,25 e 2,25 nos respectivos tratamentos. Estes melhores resultados podem ser atribuídos a alta taxa de renovação de 
água, o que não ocorreu no presente estudo. No entanto, a renovação constante de água tende a gerar grandes volumes de efluentes, os quais devem ser tratados para serem lançados em corpos receptores, e em sistemas de altas taxas de renovação de água, isto é mais difícil de realizar. Além disso, existe um alto custo adicional para manter o sistema funcionando continuamente. Quanto à sobrevivência, os autores também observaram taxas superiores a $91 \%$, semelhantes às encontradas no presente trabalho.

Em relação à taxa de conversão alimentar, os valores aumentaram com o aumento da densidade de estocagem dos alevinos, variando de 1,72 a $3,22 \mathrm{~g} \mathrm{~g}^{-1}$ (Tabela 1). Bailey et al. (2000) encontraram valores variando de 1,17 a $1,33 \mathrm{~g} \mathrm{~g}^{-1}$ nas densidades estudadas, já
Silva et al. (2002) relataram FCA de 1,22 a 1,25. Maeda et al. (2006) descreveram valores que variaram de 1,30 a 1,56. Todos estes valores encontrados foram bem melhores do que os observados nas densidades de 0,15 e 0,20 alevinos $L^{-3}$, no entanto foram próximos aos encontrados na densidade testada de 0,10 alevinos $\mathrm{L}^{-3}$, evidenciando novamente a melhor eficiência neste tratamento no presente trabalho.

A evolução do peso médio dos alevinos (Figura 1), após 15 dias de cultivo, não apresentou diferença significativa entre os três tratamentos $(\mathrm{p}>0,05)$, sendo evidenciado um melhor resultado para o cultivo realizado com a densidade de 0,10 alevinos $\mathrm{L}^{-1}$, a qual obteve maior peso médio dentre os tratamentos $(\mathrm{p}<0,05)$.

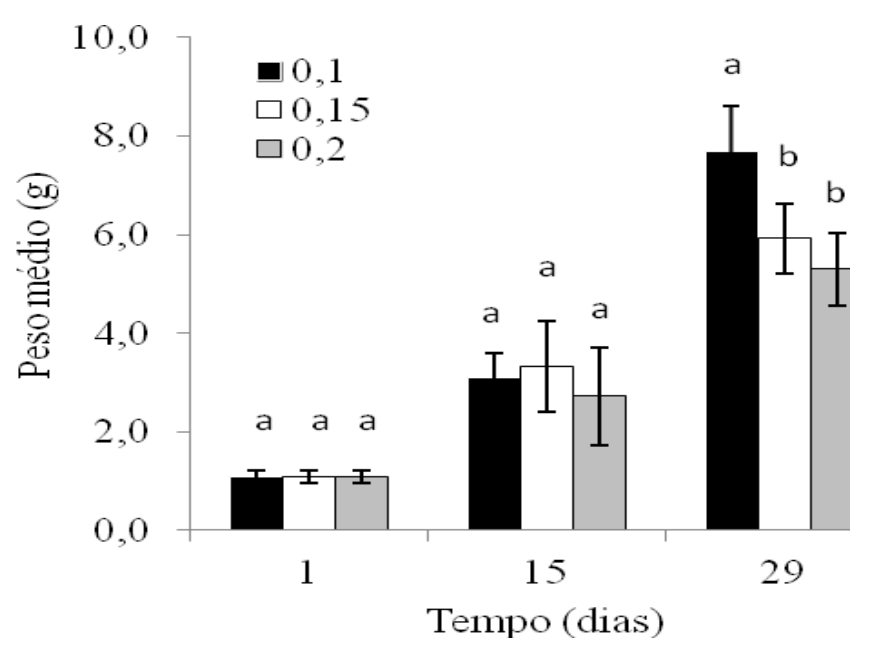

Figura 1. Evolução do peso médio dos alevinos de tilápia do Nilo cultivada com densidades de $0,10,0,15$ e 0,20 alevinos $\mathrm{L}^{-1}$. Cada ponto representa a média de quatro repetições \pm desvio padrão

Quanto aos parâmetros de qualidade de água, a temperatura é um dos parâmetros de fundamental importância em cultivos de organismos aquáticos, pois pode influenciar no aumento ou diminuição do metabolismo dos peixes (MARQUES et al., 2004). A temperatura da água de todos os cultivos se manteve semelhante $(p>0,05)$ devido às condições controladas no laboratório, não sofrendo 
Rev. Bras. Saúde Prod. Anim., Salvador, v.15, n.1, p.149-159 jan./mar., 2014 http://www.rbspa.ufba.br ISSN 15199940

influências externas. Assim como a temperatura, a salinidade, $\mathrm{pH}$ e OD também se mantiveram em níveis aceitáveis (KUBITZA, 2000) e sem diferença estatística entre os tratamentos $(\mathrm{p}>0,05)$ durante todo o período experimental (Tabela 2).

Tabela 2.Parâmetros de qualidade de água dos cultivos de tilápia do Nilo cultivada com densidades de $0,10,0,15$ e 0,20 alevinos $\mathrm{L}^{-1}$

\begin{tabular}{lccc}
\hline \multirow{2}{*}{ Parâmetros } & \multicolumn{3}{c}{ Tratamento $\left(\right.$ Alevinos $\left.{ }^{-1}\right)$} \\
\cline { 2 - 4 } & 0,10 & 0,15 & 0,20 \\
\hline Temperatura $\left({ }^{\circ} \mathrm{C}\right)$ & $28,4 \pm 0,4^{\mathrm{a}^{\mathrm{a}}}$ & $29,0 \pm 1,8^{\mathrm{a}}$ & $28,9 \pm 1,5^{\mathrm{a}}$ \\
Salinidade $\left(\mathrm{g} \mathrm{L}^{-1}\right)$ & $10 \pm 1,0^{\mathrm{a}}$ & $10 \pm 1,0^{\mathrm{a}}$ & $10 \pm 1,0^{\mathrm{a}}$ \\
$\mathrm{pH}$ & $8,3 \pm 0,4^{\mathrm{a}}$ & $8,4 \pm 0,3^{\mathrm{a}}$ & $8,6 \pm 0,2^{\mathrm{a}}$ \\
Oxigênio dissolvido $\left(\mathrm{mg} \mathrm{L}^{-1}\right)$ & $5,5 \pm 1,3^{\mathrm{a}}$ & $5,1 \pm 1,4^{\mathrm{a}}$ & $4,6 \pm 1,4^{\mathrm{a}}$ \\
\hline *Os valores representam as médias e desvio padrão dos parâmetros na água de cultivo ao longo do \\
experimento. \\
Letras diferentes representam diferença estatística ( $\mathrm{p}<0,05)$.
\end{tabular}

Estes resultados foram importantes, principalmente quanto ao OD, considerado uma das mais críticas variáveis, por afetar diretamente a resistência e sobrevivência dos peixes. Concentrações de oxigênio dissolvido menores que $4,0 \mathrm{mg} \mathrm{L}^{-1}$ podem levar a uma redução no consumo alimentar e, consequentemente, à queda no ritmo de crescimento (SARAIVA et al., 2009).

Quanto a salinidade, antes do início do experimento a mesma foi elevada através do uso de cloreto de sódio $(\mathrm{NaCl})$ e foi mantida constante, durante todo o cultivo, pela adição de água doce devido a tendência de aumento por conta da evaporação da água. As tilápias possuem a capacidade de suportar amplas variações de salinidade (eurialina), devido também a sua rusticidade, característica que confere a capacidade de adaptação a ambientes adversos, podendo se sobreviver em água doce, salobra ou salgada (KUBITZA, 2000).

As médias das concentrações de amônia, durante o período experimental, foram $0,26 \pm 0,11,0,27 \pm 0,09$ e $0,33 \pm$
$0,08 \mathrm{mg} \mathrm{L}^{-1}$ para as densidades de 0,10 , 0,15 e 0,20 alevinos $\mathrm{L}^{-1}$, respectivamente, assim, independente da densidade de cultivo, não foram observadas diferenças significativas $(p>0,05)$ nos teores de amônia entre os tratamentos antes da transferência da água para os cultivos de $S$. platensis (Figura 2, A).

Os valores de amônia, que permaneceram abaixo de $2,0 \mathrm{mg} \mathrm{L^{-1 }}$, valor recomendado por Arana (2004), podem ser explicados possivelmente devido ao consumo pela $S$. platensis e alta concentração de OD em todos os tratamentos, o que possibilitou que a amônia, provinda das sobras de ração e da excreção dos peixes, sofresse $o$ processo de volatilização e de nitrificação, ocorrendo oxidação para nitrito e posteriormente para nitrato. Isto foi evidenciado principalmente pelo aumento da concentração de nitrato e ligeiro aumento de nitrito nessa etapa do experimento, uma vez que o nitrito é a forma intermediária do processo de formação do nitrato. 

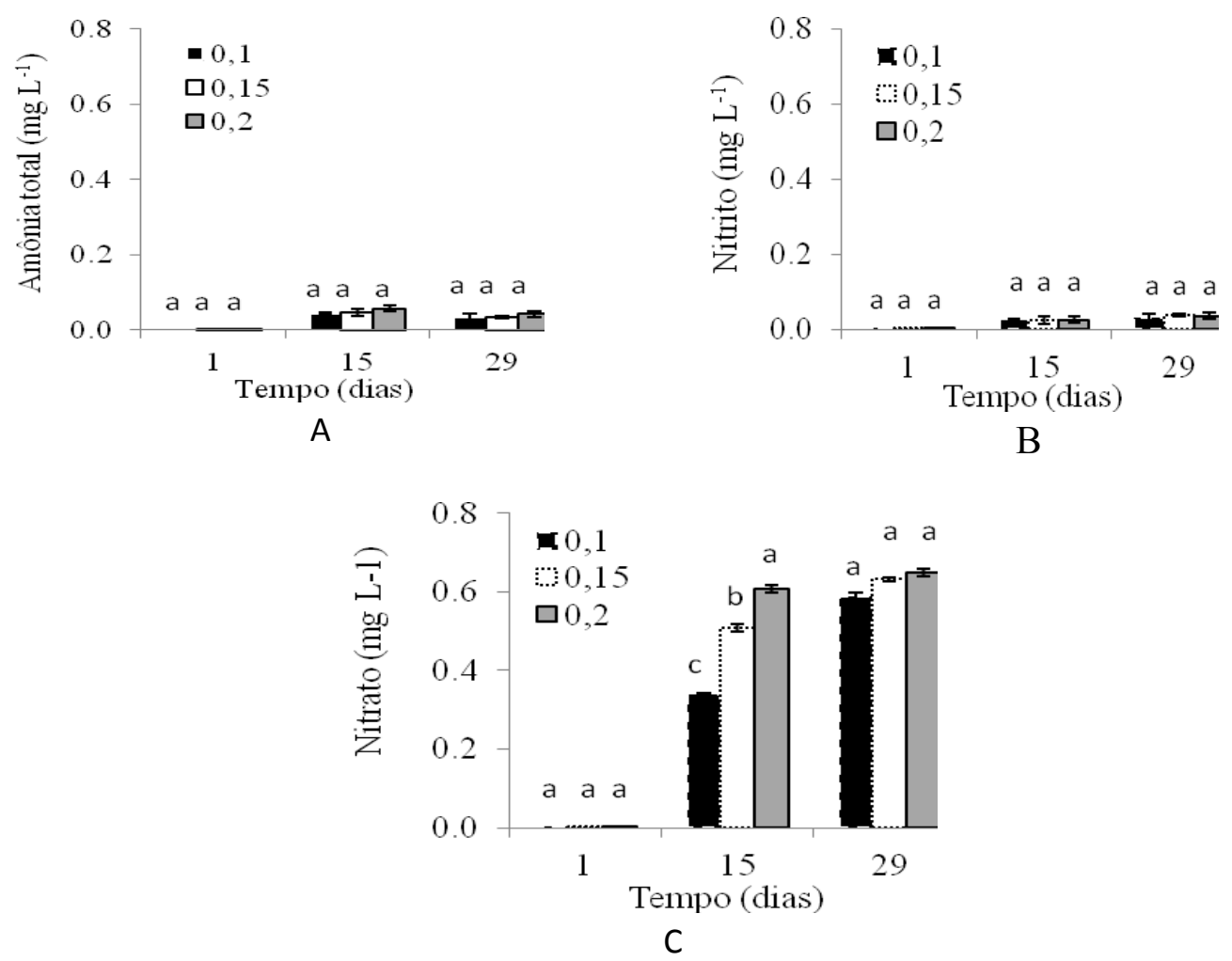

Figura 2. Concentrações de amônia (A), nitrato (B) e nitrito (C) registradas nos tanques estocados com tilápia do Nilo nas densidades de 0,10, 0,15 e 0,20 alevinos $\mathrm{L}^{-1}$. Cada ponto representa a média de quatro repetições \pm desvio padrão

Pereira \& Lapolli (2009), após cultivarem tilápia do Nilo em efluente doméstico, observaram alguns parâmetros ambientais para avaliação da qualidade do efluente. Foi também observado pelos autores que a amônia foi nitrificada a nitrito e nitrato, evidenciado incremento de nitrito de 0,1 para $2,0 \mathrm{mg}^{-1}$ e nitrato, de 0,2 para $1,5 \mathrm{mg} \mathrm{L}^{-1} \mathrm{e}$ diminuição da amônia de 19,7 para $1,3 \mathrm{mg} \mathrm{L}^{-1}$.

As concentrações de nitrito não diferiram entre os tratamentos $(p>0,05)$ e permaneceram entre 0,0 e $0,37 \mathrm{mg} \mathrm{L}^{-1}$, e a salinidade em torno de $10 \mathrm{~g} \mathrm{~L}^{-1}$, possivelmente $\mathrm{o}$ nitrito não afetou $\mathrm{O}$ desempenho zootécnico dos alevinos, já que a toxidez por nitrito pode ser minimizada com o aumento na concentração de íons cloretos $\left(\mathrm{Cl}^{-}\right)$na água, pois o nitrito e o íon cloreto competem pelo transportador de cloreto nas células das brânquias dos peixes.

Assim, peixes de água doce expostos ao nitrito tendem a diminuir a concentração de cloreto no plasma (AGGERGAARD \& JENSEN, 2001), gerando a metahemoglobina que é formada pela ligação do nitrito com a hemoglobina, conferindo a coloração marrom ao sangue, pois a metahemoglobina não consegue transportar o oxigênio, resultando em hipóxia tecidual, além disso, também provoca alterações hepáticas, e tem um efeito vasodilatador (COSTA, 2002; SÁ, 2012).

Em relação às concentrações de nitrato, observa-se que foi o composto nitrogenado que mais se acumulou 
durante $\mathrm{o}$ período experimental, variando de 0,0 no início até $6,32 \mathrm{mg} \mathrm{L}^{-1}$ para a maior densidade de estocagem, no final de 29 dias de cultivo. Houve diferença significativa entre as concentrações de nitrato nos cultivos com 0,10 alevinos $\mathrm{L}^{-1}$ e as demais, a partir da metade do experimento que durou até o final. Embora os maiores valores encontrados de nitrato ainda estejam dentro da faixa tolerável para tilápias (KUBITZA, 2000), certamente os peixes estocados na menor densidade estavam em uma maior situação de conforto, pois ficaram expostos a uma concentração significativamente inferior até os momentos de circulação da água. $\mathrm{O}$ consumo de amônia, nitrito e nitrato pela $S$. platensis reduziu bastante a quantidade desses constituintes no meio de cultivo. Segundo Derner et al. (2006), as microalgas possuem melhor afinidade para incorporar amônia. As concentrações iniciais dos compostos nitrogenados presentes nos cultivos de S. platensis são as mesmas expostas no gráfico 5. As taxas de remoção dos compostos nitrogenados dos efluentes por $S$. platensis podem ser observados na Figura 3.

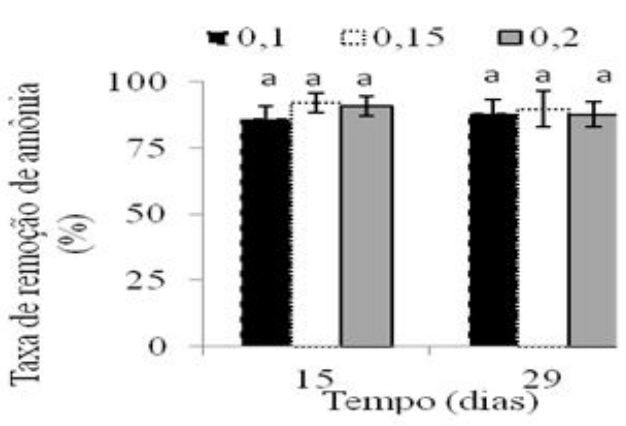

A

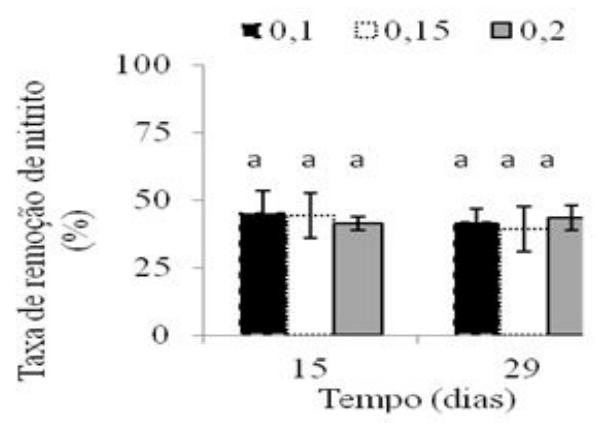

$\mathrm{B}$

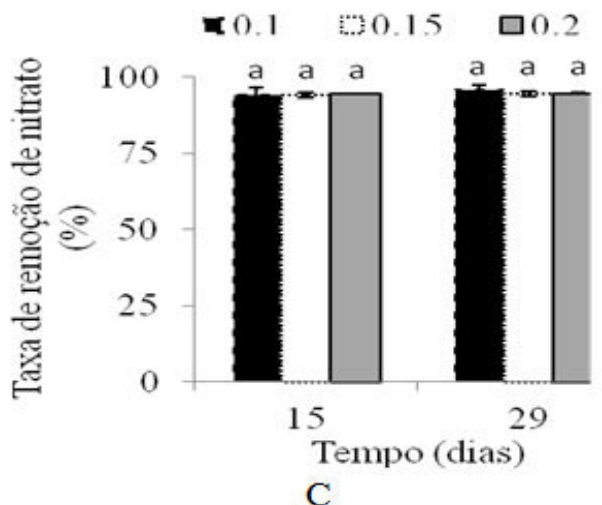

Figura 3. Taxas de remoção de amônia, nitrato e nitrito nos efluentes dos cultivos de alevinos de tilápia do Nilo nas densidades de $0,10,0,15$ e 0,20 alevinos $\mathrm{L}^{-1}$. Cada ponto representa a média de quatro repetições \pm desvio padrão

As taxas de remoção dos compostos nitrogenados foram semelhantes para amônia e nitrato $(\mathrm{p}>0,05)$ e não apresentaram diferenças significativas entre os tratamentos com diferentes densidades de estocagem. Em média, 
foram reduzidos $95,57 \pm 1,1 \%$ da amônia, $87,9 \pm 4,4 \%$ de nitrato e 42,63 $\pm 8,5 \%$ de nitrito.

Pode-se observar que as altas taxas de redução de nitrogênio por microalgas vêm se tornando uma alternativa potencial para o tratamento de efluentes, sejam industriais ou da aquicultura, o que também foi evidenciado no presente trabalho.

Kumar et al. (2010) mostraram que a microalga $C$. vulgaris removeu mais de $85 \%$ da amônia total de um efluente de suinocultura em dez dias de cultivo. Martins et al. (2007) relataram a capacidade de biorremediação da água de tanques de piscicultura pela macrófita aquática Typha dominguensis, com cinco diferentes níveis de nitrogênio $(\mathrm{N})$ em sistema fechado com uma taxa máxima de remoção de $87 \%$.

Henry-Silva \& Camargo (2008) avaliaram a eficiência das macrófitas aquáticas flutuantes Eichhornia crassipes e Pistia stratiotes no tratamento de efluentes gerados por um viveiro de piscicultura e a maior taxa de remoção foi de $34,7 \%$ para $P$. stratiotes. Bertoldi et al. (2007) avaliaram o consumo de nitrato pela microalga $C$. vulgaris em diferentes diluições de uma solução hidropônica residual. Quando os cultivos foram realizados com uma diluição de $25 \%$ as microalgas removeram $87 \%$ de nitrato do meio de cultivo, enquanto na solução original e com $50 \%$ de diluição, as microalgas consumiram 80,5 e $80,4 \%$ dos nitrato, respectivamente.

A microalga $S$. platensis demonstrou uma maior preferência para assimilar os nitrato e a amônia presentes nos efluentes de cultivo dos peixes. Dessa forma, o acúmulo desses nutrientes em cultivos de peixes pode ser utilizado para o crescimento de S. platensis, obtendo assim triplo benefício, o da produção de uma biomassa de alto valor protéico, a redução dos impactos da aquicultura, mediante a remoção de nutrientes dos efluentes de cultivo de peixes pela microalga e, ainda, o reuso da água de acordo, com o sistema de recirculação empregado.

Pode-se observar que quanto aos parâmetros zootécnicos dos alevinos, a densidade de estocagem mais eficiente foi a de 0,10 alevinos $L^{-1}$, pois foi a que proporcionou menor taxa de conversão alimentar e maior sobrevivência. A densidade inicial pode ter sido a principal causa do melhor desempenho, já que os parâmetros de qualidade de água analisados foram semelhantes em todas as densidades experimentadas.

Apesar das taxas de crescimento específico terem sido relativamente baixas, o presente trabalho apresentou vantagem de não haver geração de efluente o que, em grande escala, pode contribuir significativamente para redução de impactos ambientais negativos da aquicultura intensiva.

A integração da água entre as culturas de tilápia do Nilo e S. platensis através da circulação periódica durante $o$ experimento foi suficiente para manter os teores de amônia, nitrato e nitrito em níveis aceitáveis ao crescimento dos peixes através da assimilação desses compostos pela microalga S. platensis, viabilizando $o$ estabelecimento a integralização das culturas.

\section{REFERÊNCIAS}

AGGERGARD, S.; JENSEN, F.B.

Cardiovalscular changes and physiological response during nitrite exposure in rainbow trout. Journal Fish Biology, v.59, n.1, p.13-27, 2001.

ARANA, L.V. Fundamentos de aquicultura. Florianópolis: UFSC, 2004.349p. 
ASADUZZAMAN, M.; WAHAB, M.A.; VERDEGEM, M.C.J.; BENERJEE, S.; AKTER, T.; HASAN, M.M.; AZIM, M.M. Effects of addition of tilapia Oreochromis niloticus and substrates for periphyton developments on pond ecology and production in $\mathrm{C} / \mathrm{N}$ controlled freshwater prawn Macrobrachium rosenbergii farming systems.

Aquaculture, v.287, n.3-4, p.371-380, 2009.

BAILEY, D.S.; RACKOCY, J.E.; MARTIN, J.M.; SHULTZ, R.C. Intensive production of tilapia fingerlings in a recirculating system. In:

INTERNATIONAL SYMPOSIUM ON TILAPIA AQUACULTURE, 5., 2000, Rio de Janeiro. Proceedings... Rio de Janeiro: Panorama da Aquicultura, 2000. v.2, p.328-333.

BERTOLDI, F.C.; SANT'ANNA, E.; OLIVEIRA, J.L.B.; REBELO, A.M. Bioremoval of nitrogen and phosphorus from hydroponic wastewater by Chlorella vulgaris. Evidência, v.7, n.2, p.85-92, 2007.

COLLA, L.M.; REINEHR, C.O.; REICHERT, C.; COSTA, J.A.V. Production of biomass and nutraceutical compounds by Spirulina platensis under different temperature and nitrogen regimes. Bioresource Technology, v.98, n. 7, p.1489-1493, 2007.

COSTA, O.T.F.; DIANA, F.S.J.; FABIANA, L.P.M.; MARISA, N.F. Susceptibility of the Amazonian fish, Colossoma macropomum

(Serrasalminae), to short-term exposure to nitrite. Aquaculture, v.232, n.1-4, p.627636, 2002.

DERNER, R.B.; OHSE, S.; VILLELA, M.; CARVALHO, S.M.; FETT, R. Microalgae, products and applications. Ciência Rural, v.36, n.6, p.1959-1967, 2006.
EL-SAYED, A.M. Effects of stocking density and feeding levels on growth and feed efficiency of Nile tilapia (Oreochromis niloticus) fry. Aquaculture, v.33, n.8, p.621-626, 2002. FOOD AND AGRICULTURE ORGANIZATION - FAO. The State of World Fisheries and Aquaculture 2010. Rome, 2010.197p.

HENRY-SILVA, G.G.; CAMARGO, A.F.M. Treatment of shrimp effluents by free-floating aquatic macrophytes.

Revista Brasileira de Zootecnia, v.37, n.2, p.181-188, 2008.

JONES, A.B.; PERSTON, N.P.; DENNISON, W.C. The efficiency and condition of oysters and macroalgae used as biological filters of shrimp pond effluent. Aquaculture Research, v.33, p.1-19, 2002.

KUBITZA, F. Tilápia: tecnologia e planejamento na produção comercial. Jundiaí: F. Kubitza, 2000. 285 p.

KUMAR, M.S.; MIAO, Z.H.; WYATT, K.S. Influence of nutrient loads, feeding frequency and inoculum source on growth of Chlorella vulgaris in digested piggery effluent culture medium.

Bioresource Technology, v.101, n.15, p.6012-6018, 2010.

\section{LOURENÇO, S. O. Cultivo de} microalgas marinhas: princípios e aplicações. São Carlos: Rima, 2006. v.1, $588 \mathrm{p}$.

MAEDA, H.; SILVA, P.C.; AGUIAR, M.S.; PADUA, D.M.C.; OLIVEIRA, R.P.C.; MACHADO, N.P.; RODRIGUES, V.; SILVA, R.H. Effects of stocking density in the second finggrlinging of nile tilapia (Oreochromis niloticus) Raised in raceway system. Ciência Animal Brasileira, v.7, n.3, p.265-272, 2006. 
MARQUES, N.R.; HAYASHI, C.; SOUZA, S.R.; SOARES, T. Efeito de diferentes níveis de arraçoamento para alevinos de carpa-capim (Ctenopharyngodon idella) em condições experimentais. Boletim do Instituto de Pesca, v.30, n.1, p.51-56, 2004.

MARTINS, A.P.L.; REISSMANN, C.B.; FAVARETTO, N.; MARIA R.T. BOEGER, M.R.T.; OLIVEIRA, D.B. Capacidade da Typha dominguensis na fitorremediação de efluentes de tanques de piscicultura na Bacia do Iraí - Paraná.

Revista Brasileira de Engenharia Agrícola e Ambiental, v.11, n.3, p.324 330, 2007.

PEREIRA, C.M.; LAPOLLI, F.R. Criação de tilápias no efluente doméstico tratado em lagoas de estabilização.

Biotemas, v.22, n.1, p.93-102, 2009.

PORELLO, S.; LENZI, M.;

TOMASSETTI, P.; PERSIA, E.; FINOIA, M. G.; MERCATALI, I. Reduction of aquaculture wastewater eutrophication by phytotreatment ponds system II. Nitrogen and phosphorus content in macroalgae and sediment. Aquaculture, v.219, n.1-4, p.531-544, 2003.

SÁ, M.. Limnocultura: limnologia para aquicultura. Fortaleza: UFC. 218p. 2012.

SALARO, A.L.; LUZ, R.K.; NOGUEIRA, G.C.C.; REIS, A.; SAKABE, R.; LAMBERTUCCI, D.M. Effect of two stocking rates on the trairão (Hoplias cf.lacerdae) fingerlings performance. Revista Brasileira de

Zootecnia, v.32, n.5, p.1033-1036, 2003.
SANTOS, E.S.; FURTADO-NETO, M.; MOTA. S.; SANTOS, A.B.; AQUINO, M.D. Cultivo de tilápia do Nilo em esgoto doméstico tratado, com diferentes taxas de alimentação. Revista DAE, v.180, p.411, 2009.

SARAIVA, K.A.; MELO, F.P.; APOLINÁRIO, M.O.; SANTOS, A.J.G.; CORREIA, E.S. Densidades de estocagem de juvenis da tilápia Oreochromis niloticus (linhagem Chitralada) cultivados em tanques-rede. Revista Brasileira de Saúde e Produção Animal [online], v.10, n.4, p.963-969, 2009.

SILVA, L.B.; BARCELLOS; L.J.G.; QUEVEDO, R.M.; SOUZA, S.M.G.; KREUTZ, L.C.; RITTER, F.; FINCO, J.A.; BEDIN, A.C. Alternative species for traditional carp polyculture in southern South America: Initial growing period. Aquaculture, v.255, n.1-4, p. 417-428, 2006.

SILVA, P.C.; KRONKA, S.N.; TAVARES, L.H.S.; SOUZA, V.L. Productive performance of the nile tilapia (Oreochromis niloticus L.) in tanks with different water exchanges and stocking density in raceway Acta Scientiarum, v.24, n.4, p.935-941, 2002.

SOUZA, S.M.G.; OLIVEIRA, D.; SANTOS, C.V.; GOMES, M.E.C.; ESTEVES, C.D. Growth and feed utilization by juvenile grass carp (Ctenopharyngodon idella) feed with Azolla filiculoides) with low lipid diet. Ciências Agrárias, v.29, n.2, p.459-464, 2008.

Data de recebimento: $24 / 09 / 2013$

Data de aprovação: 21/02/2014 